\title{
Autistic expertise: A critical reflection on the production of knowledge in autism studies
}

Damian E M Milton

This article was published in Autism on 17 Mar 2014, available online:

https://uk.sagepub.com/en-gb/eur/journal/autism

http://dx.doi.org/10.1177/1362361314525281

\begin{abstract}
The field of autism studies is a highly disputed territory within which competing contradictory discourses abound. In this field, it is the voices and claims of autistic people regarding their own expertise in knowledge production concerning autism that is most recent in the debate, and traditionally the least attended to. In this article, I utilise the theories of Harry Collins and colleagues in order to reflect upon and conceptualise the various claims to knowledge production and expertise within the field of autism studies, from the perspective of an author who has been diagnosed as being on the autism spectrum. The notion that autistic people lack sociality is problematised, with the suggestion that autistic people are not well described by notions such as the 'social brain', or as possessing 'zero degrees of cognitive empathy'. I then argue, however, that there is a qualitative difference in autistic sociality, and question to what extent such differences are of a biological or cultural nature, and to what extent interactional expertise can be gained by both parties in interactions between autistic and non-autistic people. In conclusion, I argue that autistic people have often become distrustful of researchers and their aims, and are frequently frozen out of the processes of knowledge production. Such a context results in a negative feedback spiral with further damage to the growth of interactional expertise between researchers and autistic people, and a breakdown in trust and communication leading to an increase in tension between stakeholder groups. The involvement of autistic scholars in research and improvements in participatory methods can thus be seen as a requirement, if social research in the field of autism is to claim ethical and epistemological integrity.
\end{abstract}

Keywords: Autism, expertise, knowledge production, somatic affordance, tacit knowledge

\section{Introduction}

Reflecting upon investigations carried out during doctoral research that concerned the discourses produced by various stakeholders regarding the education of autistic people, a range of views and opinions was being voiced by practitioners, academics from various disciplines, parents and autistic people themselves, and some clear trends have emerged (Milton, 2011, Milton, 2012c). These trends indicate a wide divide in outlook between the various personal deficit models of autism often favoured by practitioners and certain academics (Frith, 1989; Happe, 1994), and a more sociologically situated model of autism often followed by autistic people themselves (Arnold, 2010; Graby, 2012, Milton, 2012b).

The field of autism studies is a highly disputed territory with competing contradictory discourses abounding. Within this disputed territory, however, it has been the voice and assertion of autistic people themselves to claims of expertise in knowledge production that is 
most recent in the debate, and traditionally the least listened to (Milton and Bracher, 2013). This article utilises the theories of Harry Collins and his colleagues (Collins, 2004, 2010, 2011; Collins et al., 2006; Collins and Evans, 2007) in order to reflect upon and conceptualise the various claims to knowledge production and expertise within the field of autism studies.

The article proceeds in six sections. The section 'A framework to conceptualise expertise' outlines the framework for conceptualising the acquisition of knowledge and expertise by Collins and Evans (2007). This section then introduces the term of 'interactional expertise' and asks to what extent such mutual understanding is achievable between autistic and nonautistic people. This framework was originally devised to indicate that the kind of expertise employed by specialists is often of a socially situated nature. The section 'Popular understandings' shows how such a framework can account for some of the popular understandings (and misunderstandings) that can be seen in the field of autism for those who do not have access to such specialist practices. In the section "The "machine-like" metaphor', the 'machinelike' metaphor often used to describe autistic cognition and knowledge acquisition is critiqued utilising this conceptual framework, and an argument put forward that autistic people (across the spectrum) are indeed social beings, albeit perhaps a more idiosyncratic or outsider social experience and expressions of social agency. This argument is continued in the section 'Autism and the acquisition of tacit knowledge', which explores in depth the ways in which knowledge can be acquired, and how autistic people cannot be said to totally lack such forms of knowledge. This section finishes with a critique of educational methods for autistic people that break down social information into explicit 'rules'. The section 'How does one know when interactional expertise with autistic culture has been acquired?' asks the important question of how one is to know when one has gained interactional expertise with autistic people and their culture, seen by Collins and Evans (2007) as a minimal requirement for social scientific research on cultural groups. This section reviews the use of the 'imitation game' as devised by Collins and Evans (2007), an adaptation of the Turing Test, where the 'judge' has to decide between two people, one who is a genuine member of a cultural group and who is an imposter. If such a game were to be played by social researchers in the field of autism studies, it would give an indication as to the level of interactional expertise gained, and whether interpretations by said researcher are likely to be relevant and accurate. The section 'Lost in translation?' asks whether or not due to embodied differences between autistic and nonautistic people, whether some level of understanding will always be 'lost in translation', and whether the 'double empathy problem' (Milton, 2012b) can be reduced and to what extent. The article finishes with some final remarks regarding the need for the involvement of autistic scholars in social research regarding autistic people, the increased use of participatory methods, and sets a challenge for social researchers working in this field.

\section{A framework to conceptualise expertise}

In recent decades, scholars in social theory have developed increasingly more sophisticated accounts of the nature of scientific expertise. This work explains how different claims to knowledge are defended by reference to different sorts of specialism or expertise. Collins and Evans (2007) in Rethinking Expertise set out a 'periodic table of expertise' based on the notion of socially located domains of tacit knowledge, that is, knowledge that cannot easily be made explicit or codified. This framework being predicated on a conceptual working model of loose boundaries between the categories stated. This conceptualisation was taken 
further in Tacit and Explicit Knowledge, in which Collins (2010) provides a conceptual language with which to analyse the acquisition of knowledge.

Collins and Evans (2007) suggest that expertise is primarily based on the acquisition of tacit knowledge. In theorising tacit knowledge, they distinguish between ubiquitous and specialist expertise. Ubiquitous expertise includes an endless number of skills and knowledge that sustain the forms of life and culture of society (e.g. fluency in natural language or moral sensibility), whereas specialist expertise requires immersion in the language and practice of expert communities. Collins and Evans (2007) rank the acquisition of specialist knowledge on a scale of expertise running from 'beer-mat knowledge', through 'popular understanding', and 'primary source knowledge', all being knowledge which can be acquired from a base of ubiquitous tacit knowledge, before attaining 'interactional' and finally 'contributory' expertise, which depend on the acquisition of specialist tacit knowledge.

The differences between these categories of expertise are subtle, however, and relate to the availability of socialisation into practice communities. Specialist expertise can therefore be seen as a socially restricted form of socialisation (i.e. cultural learning dependent on being embedded in a specialist social practice), while the other forms of ubiquitous expertise are said to be available to all. When one applies such categorisations to the knowledge and expertise displayed by autistic people, things tend to look a bit different. Specialist expertise can certainly be found, not least with autistic culture, community and language (Arnold, 2010; Lawson, 2008; Sinclair, 1993, 1999), yet more common forms of ubiquitous expertise are often deemed to be deficient or lacking due to cognitive deficit, such as the ability for 'dynamic thinking' (Gustein, 2000), or 'cognitive empathy' (Baron-Cohen, 2012).

Following Wittgenstein, Collins and Evans (2007) argue that it is in the use of a concept which determines its meaning, thus understanding specialist meaning takes immersion in a way of life, rather than information gathering alone. In acquiring initial 'beer-mat' knowledge or simple popular understandings, such deep immersion is not required, and as such the specialist ubiquitous expertise of 'ordinary people' should not be confused with the expertise of technical specialists within a particular domain of knowledge. The amount of interactional expertise achievable between autistic and non-autistic people, or how much these barriers are cultural, biological or whether such a distinction can be made (Hacking, 2009) has not been adequately 'answered' or investigated through a sociological or contextualised lens in sufficient depth. As will be returned to later in this article, however, I argue that some level of interactional expertise must be possible, as no autistic person is completely uncommunicative. The interactional expertise shown by non-autistic social researchers is, however, often clearly insufficient, given the criticisms made of such investigations by autistic scholars (Arnold, 2012a; Milton and Bracher, 2013). Gaining expertise in what it is to be autistic would take immersion in the culture and practices of autistic people, yet it is questionable as to what extent such immersion is possible for non-autistic people and it is certainly doubtful that many established scientists have made the effort. Yet, for Collins and Evans (2007), interactional expertise should be seen as a basic standard for social scientific research. Such a gap in understanding can be seen as adding to the distrust and offence taken by autistic people to how 'we' as a group are examined, inspected and interpreted by those of a non-autistic dispositionality; as Moon, a neurodiverse activist has stated, being 'fishbowled' (Milton and Moon, 2012a). It should also be remembered who the 'contributory experts' are, regarding the creation of autistic subjectivity and culture in the first place: 
autistic people themselves. Interactive expertise does not make someone an autistic person contributing to autistic culture, but someone more able to engage and interact with autistic language and communications. The involvement of autistic scholars in research and improvements in participatory methods can thus be seen as a requirement, if social research in the field of autism is to claim ethical and epistemological integrity.

Claims to knowledge are of course linked to power and social closure (the restricting and privileging of social opportunities for one group of people at the expense of another) which influence the social valuing of various forms of specialist expertise (Rose, 1999). It is sometimes suggested in the case of autistic people, moreover, that an apparent inability to acquire basic forms of ubiquitous expertise lead autistic people to be unable to develop specialist expertise, even with reference to their own subjectivity and social positionality (e.g. Hendriks, 2012). Instead, expertise that is remarked upon as being achievable by some autistic people is seen in much non-autistic literature on the subject as residing in some kind of exotic other, within a discourse of the extraordinary autistic 'savant' (Arnold, 2012b). Such a lack of social expertise and understanding has been called into question, however, by the initial emergence of autistic autobiographies, followed by autistic scholarship and the rise of advocacy organisations run by autistic people themselves (Autistic Rights Group Highland (ARGH), 2013; Autonomy, 2013; Autscape, 2013). It is of course true that not all autistic people are capable of such expressiveness, but a number of autistic people thought to have been incapable of communicating have found ways in which to do so (Wurzberg, 2011).

\section{Popular understandings}

Due to the specialised nature of expertise, Collins and Evans (2007) suggest that scientists must simplify their work in order to explain something of it to a professional audience, and even more for popular audiences:

'...in the case of disputed science, a level of understanding equivalent to popular understanding is likely to yield poor technical judgements.' (Collins and Evans, 2007: 21).

Popular understandings can lead to polarised reactions to scientific research. This can sometimes involve accepting a simplified version of evidence as truth and fact. We see this, for example, in the way that the idea of there being a theory of mind deficit in autism is now commonly depicted as 'factual' in popular literature, despite it being more accurately described as a working model or partial heuristic in the scientific world. Or it can involve the acceptance of claims without due thought. This is witnessed in the promotion of false conspiracy theories, as in the moral panic regarding vaccines and autism. For Collins and Evans (2007), such misinterpretations are strengthened by media representations of disputed science as something more revealing of knowledge being produced within a community of experts. One could also say, however, that popular misunderstandings are exacerbated by the claims of some academics themselves. The use of crude terms such as an 'extreme male brain' with reference to autism in the scientific literature could clearly help produce unsurprisingly simplistic understandings outside the scientific community (BaronCohen, 2008).

\section{The 'machine-like' metaphor}

The tendency discussed earlier for the scientific community to neglect the specific expertise of the autistic community is further exacerbated by the widely prevailing notion that autistic 
people are somehow 'machine-like' or lacking in the socialisation necessary for effective communication. Asperger (1991) himself wrote that:

'The autist is only himself ... and is not an active member of a greater organism which he is influenced by and which he influences constantly.' (Asperger, 1991: 38)

Approaches to the ontology of autism have been evolving ever since the phenomenon came into the clinical lexicon, yet the notion of the autistic person somehow being 'machine-like', incapable of true socialisation, possessing 'zero degrees of empathy' or having an impairment in their 'social brain' (Baron-Cohen, 2012), has remained a repeated descriptive metaphor (Hendriks, 2012).

Collins (2010) utilises the notion of strings as building blocks of signs and symbols, a physical object that only has an effect due to what happens to it. While language is conceptualised as a set of meanings located in society, strings are conceptualised as base objects that are the means by which language is inscribed and shared. Collins (2010) suggests that computers deal in strings and not language interpretation. No string has inherent meaning, as it is in linguistic interpretation that meaning is constructed and negotiated. Autistic people are often misinterpreted as only being able to deal in strings of information, as though they totally lacked a sociality and a language, or as though lacking meaning-making emotions.

A recent example of this phenomenon is seen within the theory and practice of Relationship Development Intervention (Gustein, 2000), where autistic people are depicted as lacking 'dynamic thinking' and possessing instead a strength in 'static thinking' (in other words, processing strings of information). Yet, autistic artwork (Mullin, 2009) or the 'non-verbal' language expressed by

Amanda Baggs (2007) is not reducible to strings alone in terms of how they are produced, but an engagement with the collective lifeworld of social life (i.e. learnt through some kind of sharing of social experiences).

The implications of this prevailing misunderstanding of autistic socialisation are extensive. Collins and Evans (2007) suggest that there has been a general move towards seeing knowledge and ability as concerning embodied experience or else competence, that is, what one 'does' is what matters rather than just what someone can 'reproduce' (cited in Collins and Evans, 2007). For Collins and Evans (2007), moreover, the primary site of the acquisition of knowledge and expertise is social, thus the mastering of a skill requires more than the embodiment of it, but the socialisation of people into relevant social practices. This, they say, is the difference between being able simply physically to balance on a bike and actually being able to negotiate traffic. Collins and Evans (2007) argue that polymorphic actions (actions that depend on context for interpretation and continuation and thus not reproducible by machines) require social understanding and flexibility to adapt actions to changing social contexts. This is the reason given by Collins and Evans (2007) to suggest why machines cannot replicate humans. If one is to believe current dominant ideologies regarding what autism is, then, it could also be argued that autistic people are machine-like, and unable to replicate appropriately the behaviours and understandings of non-disordered humans (at least without 'intervention'). There is clearly a need to correct this ideological error. Although processing of strings of information can be a strength found among many on 
the autism spectrum (Murray et al., 2005), not all autistic displays of knowledge and expertise can be explained away as highly honed mimeomorphic actions (actions performed in the same way each time and thus can be reproduced mechanically). With autistic people, especially those who acquire verbal articulacy, one often finds the sociality of an 'outsider' (Becker, 1963), an alienated social identity, but a social identity nonetheless. Also, it is often said that one of the most defining features of autism is a 'spiky' cognitive profile (Milton, 2012c) that can lead to extreme strengths in areas of mimeomorphic actions, but also potentially a widening of perspective and a distinctive kind of sociality, particularly in later years of development. Such a sociality is often stigmatised (Milton, 2013b) rather than being seen as a potential asset within communities of practice.

\section{Autism and the acquisition of tacit knowledge}

What the argument so far demonstrates is that it is necessary to develop a clear account of the kinds of knowledge that autistic people may be able to acquire and to reject misplaced assumptions which prevent us from doing just that. In his conception of the acquisition of tacit knowledge, Collins (2010) demarcates three main categories: weak/relational, medium/somatic and strong/collective. Weak relational tacit knowledge arises from social interaction, yet any piece of it in principle can be made explicit. Collins (2010) gives the example of an experienced warehouseman who can find specific objects within a warehouse, yet would not be able to list every object; however, one could program a computer to do just this task. At times, knowledge that can be made explicit is not due to accident, lack of time or, as Collins (2010) indicates, 'mismatched saliences'.

For Collins (2010), somatic tacit knowledge is knowledge that can be explained or written down but cannot be used in a pragmatic way by humans due to the limitations of their bodies (their 'somatic affordances'). Such knowledge can in principle be made explicit, yet may be very difficult to do so. Machines of the right design may be able to apply such knowledge due to differing somatic affordance (e.g. machines can compute mathematical sums far quicker than humans can). Somatic-affordance tacit knowledge can only be performed and applied as actions in practice due to the affordances of the materials from which a body or entity is made. In this respect, the limit is not explicability but embodiment. Strong or collective tacit knowledge, as defined by Collins (2010), can only be acquired through immersion in the language and practices of society and is conceptualised as a property of society. In this conceptualisation, individuals are seen as 'parasitic' on the body of social knowledge (or needing to draw upon social experiences to form tacit knowledge). This ability to be 'parasitic' on the social body is a unique property of humans, as neither other animals nor machines are capable of such collective knowledge acquisition. The term used by Collins (2010) to identify this split is 'Social Cartesianism'.

An interesting example utilised by Collins (2010) is the description of a scene from Star Trek: The Next Generation regarding the character of Lt Commander Data, a character often described as being representative of an autistic persona. In the scene, Data was able to learn dance steps to a routine without difficulty, and then in time was able to improvise dance steps of his own. Collins (2010) rightly points out that a machine would not be capable of improvisation. Yet, as an example, if I were to be placed in such a situation, it would be virtually impossible for me to enact a set dance routine, but I would have no trouble at all in improvising one, whether such a dance would win any awards in the eyes of others is debatable though. Collins (2010) points out that domesticated animals, while immersed in 
human society, are not able to be socialised, in the sense one does not encounter vegetarian, arty or nerdy dogs, they are simply just dogs. This sociality is often said to be impaired in autistic people, even mentioned by Collins (2011) when using as an example of the autistic savant who appears to manage their performances in the absence of collective tacit knowledge, with this absence seeming to be a principal feature of the autistic lifeworld. Yet, one does encounter autistic people who are vegetarian, artistic and certainly nerdy. Autistic people have distinct interests and abilities that involve social practices, and this includes those who are deemed 'non-verbal', who are often musical or artistic and whose bodily movements have been argued to be a form of language (Baggs, 2007, Milton, 2013a).

If autistic people are primarily machine-like, then where do the idiosyncratic expressions of autistic people (Mullin, 2009) originate? If one were to follow the theory of Murray et al. (2005), perhaps it is the affordances of an autistic mind-set leading to the honing-in on particular aspects of the social which inspire interest and attention - a monotropic social being, with a fragmented experience of the social. Indeed, if artificial intelligence is one day produced, it may perform similarly to an autistic person, but this performance may contain a liking for unusual improvisation:

'What is being argued is that humans differ from animals, trees, and sieves in having a unique capacity to absorb social rules from the surrounding society - rules that change from place to place, circumstance to circumstance, and time to time.' (Collins, 2010: 124).

Autistic people are often taught social 'rules' as if they are more fixed and static than they actually are in lived reality, which only causes more confusion. Much social skill or behavioural training with autistic people is predicated upon breaking down such information into explicated strings of information, which does little to help autistic people adjust to the changing flux of negotiated socially constructed realities (Milton, 2012b). By the use of such methods, autistic people have their fragmented social perceptions reinforced via the very way 'social skills' are being taught. Recently, however, methods such as Intensive Interaction (Nind and Hewitt, 1994), which focus on relationship building and child-led activities, have begun to challenge this dominance within the field.

\section{How does one know when interactional expertise with autistic culture has been acquired?}

Following the argument presented here, one of the primary tasks facing those attempting to work with autistic people or to analyse the nature of autism is to appreciate the distinctive knowledge autistic people possess and to build more constructive ways of relating to it. As Hacking (2009) puts it:

'They are creating the language in which to describe the experience of autism, and hence helping to forge the concepts in which to think autism.' (Hacking, 2009: 1467)

Over the past two decades, there has been the growth of a variety of autistic communities, both online and in conferences run by and for autistic people, such as Autscape (2013). Following the insights of Collins and Evans (2007), in order to study such a social group, interactional expertise is needed. The amount of interactional expertise and understanding of autistic culture exhibited by social researchers in the field, however, has often left a great deal to be desired (Arnold, 2012a). Misrepresentations of autistic culture are widespread 
within current literature and have hampered progress in the field (Milton and Bracher, 2013). When autistic people have been involved in research, this has often added much to the work produced, as the recent work of the Autism Education Trust demonstrates (Wittemeyer et al., 2011; Wittemeyer et al., 2012).

Collins (2004) argues that interactional expertise can simply be seen in the ways an individual can interact within a practice community, and that it is a quality utilised by specialist journalists and championed by interpretive social scientific methods (Collins et al., 2006), a form of realist pragmatic 'verstehen' (an understanding of the intentions and motives behind the actions of others). In order to test the level of interactional expertise one has with an outsider group to one's own, Collins et al. (2006) devised an alternative to the Turing test, known as the 'imitation game'. Collins et al. (2006) suggested that imitation games investigate the specific linguistic abilities of interactional experts, contributory experts and non-experts. These experiments utilise three computers linked via a wireless network with specialist software. Judges who are contributory experts type questions to the other participants, probing for possession of expertise. One participant is genuine and the other is someone without contributory expertise. After each question, the judge makes a guess with a confidence level associated with the guess. The session continues until the judge feels there is nothing left to be gained by continuing. Interactional expertise is purported to be demonstrated whenever the proportion of correct guesses is greater than that which would have been achieved by chance alone. The confidence levels of guesses are split into the following four levels:

Level 1: I have little or no idea who is who.

Level 2: I have some idea who is who - but I am more unsure than I am sure.

Level 3: I have a good idea who is who - and I am more sure than unsure.

Level 4: I am pretty sure I know who is who.

Guesses at level 3 or 4 of confidence are scored as either correct or incorrect, while all guesses right or wrong at levels 1 and 2 are counted as being uncertain. Whenever judges change their level of confidence, they are asked as to why this is the case. A second phase to the test is then to send the dialogues contained to a set of more judges.

Imitation game experiments conducted by Collins et al. (2006) show that those well socialised into the language of a specialist group are linguistically indistinguishable from those with full practical socialisation, yet distinguishable from those who are not well socialised into such specialised discourses. Thus, tacit knowledge of specialist languages can be acquired without having the tacit knowledge associated with the practices of specialist cultures to the level of contributory expertise.

If one were to apply the methodology of imitation games within the field of autism studies, a number of issues would need to be taken into account, such as general linguistic ability, potential personal connections between judge and participant, asking autistic people to potentially lie and so on. Having said this, they would no doubt yield interesting results. Would academics, practitioners and even parents be able to pass such a test in pretending to be autistic? Would social theorists who write about the autistic community and the neurodiversity movement be able to pass as a self-advocate? Such a test would give some 
indication as to the level of interactional expertise gained, and whether interpretations of autistic communities and culture can be said to be relevant and accurate depictions.

\section{Lost in translation?}

Interactional expertise can be seen as an important step towards mutual understanding, yet in the case of interactions between autistic and non-autistic people, is something always going to be 'lost in translation'? When differences in disposition and social understandings have foundations in neurological diversity, how much interactional expertise is possible? Is some level of expertise in what it is to be autistic on a phenomenological level of lived experience always beyond the grasp of non-autistic social scientific researchers? It could be said that autism is a state-specific expertise (Collins and Evans, 2007). Is the somatic affordance (the limitations of one's physiology) of an autistic bodily state a necessary condition for interactional expertise regarding autistic subjectivity? If this is the case, then interactional expertise between autistic and non-autistic people would always be constrained and partial at best. Both the philosophers Nagel (1974/1981) and Wittgenstein (cited in Collins and Evans, 2007) wrote thought experiments regarding the possibility of understanding what it would be like to be an animal (other than human). Nagel (1974/1981) asked the question 'What is it like to be a Bat?', while Wittgenstein (cited in Collins and Evans, 2007) asked the question 'If a Lion could speak, would a human being be able to understand it?' The conclusions of these thought experiments were that it was impossible to conceptualise of such an 'other', and to remove oneself from one's own perceptions and somatic (embodied) limitations. So, if the somatic affordances of autistic and non-autistic people are significantly different, is understanding the autistic lifeworld in the perceptions of a non-autistic onlooker a more nuanced version of understanding the perception of a bat or attempting to speak 'Lionese' (and vice versa)? This difficulty is exacerbated by what might be called the 'double empathy problem' (Milton, 2012b). Collins (2004) wrote that:

'Certainly, almost everything I write, and that includes the straightforward pieces, seems open to astonishing misinterpretation by at least a few people.' (Collins, 2004: 105)

While Collins (2004) was referring to the way in which his own writings are misinterpreted, such a breakdown in communication is a daily experience for autistic people (Milton 2013b). The autistic form of life does not conform to assumed social normativity and does not easily extend outward into the social, leading to a 'double empathy problem' between people of diverse dispositions (Milton, 2012b), that is, both parties struggle to understand and relate to one another. Such differences in presentation can lead to dyspathic reactions (Cameron, 2012) and stigma (Milton, 2013b), often leading to ill-fated attempts at normalisation and a continuing vicious cycle of psycho-emotional disablement (Milton and Moon, 2012b).

Collins and Evans (2007) put forward a minimal embodiment thesis and social embodiment thesis to the acquisition of knowledge. Yet, when brains do operate differently and affect social embodiment, a double empathy problem ensues easily. Reaching interactional expertise may be blocked by embodied differences of somatic affordance. Yet, for all the language and rhetoric of being from a 'different planet' or feeling like an 'anthropologist on mars', autistic people remain both human and social, albeit idiosyncratically, with diverse experiences of socialisation, or the lack of access into communities of practice to be immersed in. Such a lack of access to communities of practice can also lead to social isolation and anomie and negative consequences for individual mental and physical well- 
being (Milton and Moon, 2012b). Indeed, the construction of another 'silo' (linguistic community of experts) of the neurodiversity movement can be seen as an attempt to break down the 'silo mentality' itself (Arnold, 2010, Milton, 2012d) within the study of autism. Thankfully, there are now the beginnings of a more concerted effort from researchers, both autistic and nonautistic, to improve the participatory nature of the research agenda (Milton et al. 2012; Milton and Bracher 2013; Pellicano, 2012, Pellicano et al., 2013). Yet, difficulties remain even if the effort is made.

In the field of autism studies, little produces more debate than claims as to 'who can speak for autistic people' (Pellicano, 2012; Rudavsky, 2011). All stakeholder groups make differing claims to specialist contributory knowledge, but working within differing communities of practice with differing frames of reference. One could speculate that the sociological awareness of many neuroscientists studying autism could fail at the 'beer-mat' challenge, and vice versa, given the ever-increasing specialism inherent in academic training. As Collins (2010) put it:

'Developmental psychology offers valuable insights into the processes humans go through as they become parasites on collective tacit knowledge but the explanation is far from complete.' (Collins, 2010: 145)

The mutual incomprehension that Collins (2011) talks about as a potential consequence of the lack of the centrality of language and humans as 'social parasites' is exactly what often happens in interactions between autistic and non-autistic people, even when verbal skills have been gained. In order to build bridges and practice languages, at the very least, autistic people need to be listened to (Milton et al. 2012; Milton and Bracher 2013; Pellicano, 2012; Pellicano et al., 2013):

'Indeed, it is hard to see why mutual incomprehension would not go right down to the level of individual personal experience.' (Collins, 2011: 283)

\section{Final remarks}

According to the minimal embodiment thesis of Collins and Evans (2007) regarding interactional expertise, people who cannot perform a particular task or skill, and who therefore cannot have the embodied expertise associated with it, can still talk about that skill as if they did possess the embodied skills. Interactional expertise thus raises a key question about the level of embodiment that is needed for expertise to be transferred. For proponents of the embodiment thesis, quite a lot of embodiment is required, yet from the perspective of Collins and Evans (2007), much less embodiment is generally needed:

'The claim associated with the idea of interactional expertise is that mastery of an entire form of life is not necessary for the mastery of the language pertaining to the form of life. This is a big claim and needs strong proof.' (Collins and Evans, 2007: 77)

The somatic affordance of autistic and non-autistic dispositions may well create a large double empathy problem (Milton, 2012b), where both have a difficulty in understanding the nuances of one another's perception and sociality. To what extent can anyone immerse themselves in the language and culture of the other? At least some must be the answer, as autistic people are not 'aliens' despite the popular use of the term within autistic culture. 
Such an immersion can also be said to be the inspiration of approaches to the education of autistic people that have a more child-led focus (Milton, 2012c):

'The position argued here is that you do not have to use your body (acquire contributory expertise) in order to speak the language of the domain (acquire interactional expertise)...' (Collins and Evans, 2007: 78)

This is a debatable position, however, if one considers autistic language can be 'non-verbal' and based upon bodily movements and sensations (Baggs, 2007, Milton, 2012a). In my view, the level of embodiment needed for interactional expertise with autistic people remains unanswered, yet the imitation game experiments devised by Collins and Evans (2007) may provide a starting point by which this topic could be explored further. It must also be remembered who, in this conceptualisation, are the contributory experts regarding autistic subjectivity and culture, for this does take embodiment - that is, autistic people themselves.

In the history of autism studies, expertise has been claimed by many differing academic schools of thought, practitioners, parents, quacks and so on. Yet, the one voice that has been traditionally silenced within the field is that of autistic people themselves. Due to a lack of interactional expertise with autistic communities (Arnold, 2012b; Milton and Bracher, 2013), one could say a negative spiral has ensued. Consequently, autistic people have often become distrustful of researchers and their aims and are frozen out of processes of knowledge production (Milton et al., 2012). Such a context results in a lack of interactional expertise between researchers and autistic people and a breakdown in trust and communication (Milton, 2012b) leading to an increase in tension between stakeholder groups (Milton, 2011).

Finally, it is hoped that those wanting to research the sociality of autistic people may take up the challenge of an imitation game.

\section{Acknowledgements}

The author would like to thank Harry Collins, Rob Evans and Mike Bracher for their commentary during the development of this article.

\section{Funding}

This research received no specific grant from any funding agency in the public, commercial or not-for-profit sectors.

\section{References}

Arnold $L$ (2010) The medium is the message. Available at: http://www.ucl.ac.uk/cpjh/Arnold (accessed 16 February 2012).

Arnold L (2012a) Autism (book review). Disability and Society 27(5): 729-730.

Arnold L (2012b) The social construction of the savant. In: Critical disability studies conference, Lancaster University, 
Lancaster, 12 September.

Asperger H (1991) [1944] Autistic psychopathy in childhood, translated and annotated by Frith U [1944]. In: Frith U (ed.) Autism and Asperger syndrome. Cambridge University Press, pp.37-92.

Autistic Rights Group Highland (ARGH) (2013) http://www.

arghighland.co.uk/ (accessed 5 August 2013).

Autonomy (2013) Autonomy, the Critical Journal of Interdisciplinary Autism Studies. [online]: http://www.larry-arnold.net/Autonomy/index.php/autonomy (Accessed

24 July 2013).

Autscape (2013) Finding a voice. [online]: http://www.autscape.org/ (Accessed 24 July 2013).

Baggs A (2007) In my language. [online]: http://www.youtube.com/watch?v=JnylM1hl2jc (Accessed 19 October 2012).

Baron-Cohen S (2008) Autism and Asperger Syndrome (The Facts). Oxford: Oxford University Press.

Baron-Cohen S (2012) Zero Degrees of Empathy: A New Understanding of Cruelty and Kindness. Harmondsworth: Penguin Books.

Becker H (1963) Outsiders. New York: Free Press.

Cameron L (2012) Dyspathy: The Dynamic Complement of Empathy. Milton Keynes: The Open University.

Collins H (2004) How do you know you've alternated? Social

Studies of Science 34: 103-106.

Collins H (2010) Tacit and Explicit Knowledge. London: University of Chicago Press.

Collins H (2011) Language and practice. Social Studies of Science 41(2): 271-300.

Collins H and Evans R (2007) Rethinking Expertise. London: University of Chicago Press.

Collins H, Evans R, Ribeiro R, et al. (2006) Experiments with interactional expertise. Studies in History and Philosophy of

Science 37: 656-674.

Frith U (1989) Autism: Explaining the Enigma. London: Wiley-Blackwell.

Graby S (2012) To be or not to be disabled: autism, disablement and identity politics. Paper presented at the 'Theorising Normalcy and the Mundane' conference, University of Chester, Chester, 27 June. 
Gustein S (2000) Autism Aspergers: Solving the Relationship Puzzle - A New Developmental Program That Opens the Door to Lifelong Social and Emotional Growth. Arlington, TX: Future Horizons.

Hacking I (2009) Autistic autobiography. Philosophical Transactions of the Royal Society B: Biological Sciences 364(1522): 1467-1473.

Happe F (1994) Autism: An Introduction to Psychological Theory. London: Psychology Press.

Hendriks R (2012) Autistic Company. Amsterdam: Rodopi Bv Editions.

Lawson W (2008) Concepts of Normality: The Autistic and Typical Spectrum. London: Jessica Kingsley.

Milton D (2011) Educational discourse and the autistic student: an 'inside-out' approach. In: 9th annual postgraduate education conference, University of Birmingham, Birmingham, 5 July 2010.

Milton D (2012a) Embodied sociality and the conditioned relativism of dispositional diversity. In: Theorising Normalcy

2012, University of Chester, Chester, 26 June.

Milton D (2012b) On the ontological status of autism: the double empathy problem. Disability and Society 27(6): 883-887.

Milton D (2012c) So What Exactly Is Autism? London: Autism Education Trust.

Milton D (2012d) Theorising autism. In: Theorising autism pilot day, University of Birmingham, Birmingham, 28 September.

Milton D (2013a) Clumps: and autistic reterritorialisation of the rhizome. In: Theorising normalcy and the mundane. 4th

International Conference, Sheffield Hallam University, 4 September 2013.

Milton D (2013b) 'Filling in the gaps', a micro-sociological analysis of autism. Autonomy 1(2).

Milton D and Bracher M (2013a) Autistics speak but are they heard? Medical Sociology Online 7(2): 61-69.

Milton D and Moon L (2012a) 'And that Damian is what I call life changing': findings from an action research project involving autistic adults in an online sociology study group. Good Autism Practice 13(2): 32-39.

Milton D and Moon L (2012b) The normalisation agenda and the psycho-emotional disablement of autistic people. Autonomy, the Critical Journal of Interdisciplinary Autism Studies 1(1). 
Milton D, Mills R and Pellicano $L$ (2012) Ethics and autism: where is the autistic voice? Commentary on Post et al. Journal of Autism and Developmental Disorders. Epub ahead of print 11 December. DOI: 10.1007/s10803-012-1739-x.

Mullin J (2009) Drawing Autism. New York: Mark Batty.

Murray D, Lesser M and Lawson W (2005) Attention, monotropism and the diagnostic criteria for autism. Autism

9(2): 136-156.

Nagel T (1974/1981) What is it like to be a bat? In: Hofstadter DR and Dennett DC (eds) The Mind's I: Fantasies and Reflections on Self and Soul. London: Penguin Books, pp. 391-402.

Nind M and Hewitt D (1994) Access to Communication. London: David Fulton.

Pellicano L (2012) Who Should Speak for Autistic People?

London: Institute of [online]: http://ioelondonblog.wordpress.com/2012/06/20/who-should-speakfor-autistic-people/ (Accessed 9 July 2013).

Pellicano L, Dinsmore A and Charman T (2013) A Future Shaped Together: Shaping Autism Research in the UK. London: Institute of Education.

Rose N (1999) Governing the Soul: Shaping of the Private Self. London: Free Press.

Rudavsky S (2011) Adults with autism speak out. USA Today. [online]:

http://usatoday30.usatoday.com/news/nation/story/2011-11-06/autism-adults/51089566/1 (Accessed 9 July 2013).

Sinclair J (1993) Don't mourn for us. [online]: http://www. autreat.com/dont_mourn.html

Sinclair J (1999) Why I dislike 'person-first' language. [online]: http://www.cafemom.com/journals/read/436505/ (Accessed 22 May 2013).

Wittemeyer K, Charman T, Cusack J, et al. (2011) Educational Provision and Outcomes for People on the Autism Spectrum. London: Autism Education Trust.

Wittemeyer K, English A, Jones G, et al. (2012) The Autism Education Trust Professional Competency Framework. London: Autism Education Trust.

Wurzberg G (2011) Wretches \& Jabberers. State of the Art, Inc. Available at: http://www.wretchesandjabberers.org/ (Accessed 24 January 2013). 